\title{
A LONG SURVIVAL OF A PATIENT WITH BRAIN METASTASIS OF UNKNOWN SITE OF THE PRIMARY TUMOR
}

\author{
W. Ben Kridis', *, S. Sghaier', N. Toumi', Z. Boudawara', A. Khanfir ${ }^{1}$, J. Daoud ${ }^{3}$, M. Frikha ${ }^{1}$ \\ ${ }^{1}$ Department of Oncology, Habib Bourguiba Hospital, Sfax 3029, Tunisia \\ ${ }_{2}^{2}$ Department of Neurosurgery, Habib Bourguiba Hospital, Sfax 3029, Tunisia \\ ${ }^{3}$ Department of Radiotherapy, Habib Bourguiba Hospital, Sfax 3029, Tunisia
}

\begin{abstract}
Eighty percent of brain metastases (BM) are diagnosed in patients with known primary site of cancer. BM of unknown primary represents a difficult diagnosis. In up to $15 \%$ of patients with $\mathrm{BM}$, the site of the primary tumor will not be detected despite investigations. The prognosis of this entity is very poor. We report here a case of a long survival of a patient with brain metastasis of unknown primary. The conclusion that can be drawn is that within BM of unknown primary exist patients with a very good prognosis that must be collected and published in order to base recommendations.
\end{abstract}

Key Words: brain metastases, unknown primary, prognosis.

Eighty percent of brain metastases (BM) are diagnosed in patients with known primary site of cancer. BM of unknown primary (BMUP) represents a difficult diagnosis [1, 2]. In up to $15 \%$ of patients with BM, the site of the primary tumor will not be detected despite investigations [3]. Even though the primary remains undefined during the follow up, some of these patients found to have a favorable prognosis. In fact, cancer of unknown primary (CUP) has been divided into favorable group (20\%) and unfavorable group ( $80 \%$ ) based on histopathology and clinical manifestations [2]. We report here a case of an exceptional long survival of a patient with BMUP.

\section{CASE REPORT}

A 50-year-old man was referred to our hospital in October 2002 because of increased headaches, vomiting and dizziness evolving for two weeks. Glasgow score was quoted at 14/15. Neurological examination revealed kinetic cerebellar syndrome. Fundus examination revealed a peripapillary hemorrhage in the right eye. Brain magnetic resonance imaging $(\mathrm{MRI})$ showed a $4 \times 2 \mathrm{~cm}$ lesion of the right cerebellum with a T1 weighted hyposignal, T2 weighted hypersignal and a heterogeneous enhancement. This lesion compressed the brainstem and the fourth ventricle causing a triventricle expansion with signs of active hydrocephalus (Fig. 1). The patient underwent a complete resection of the tumor. The pathologic study showed polyhedral tumor cells arranged in solid sheets with a clear abundant cytoplasm. The tumor stroma had a vascular component of endocrine type without necrosis. The antibody panel consisted of reagents directed to antigens: CK7, CK20, EMA, NSE, chromogranin, synaptophysin, glial fibrillary acidic protein (GFAP), TTF1, $A C E$ and vimentin. The immunohistochemical staining

Submitted: December 23, 2017.

*Correspondence: E-mail: walabenkridis@yahoo.fr Tel.: +21694492526

Abbreviations used: BM - brain metastases; BMUP - BM of unknown primary; CT - computed tomography; CUP - cancer of unknown primary; GFAP - glial fibrillary acidic protein; KPS - Karnofsky performance status; MRI - magnetic resonance imaging; PET - positron emission tomography; WBRT - whole brain radiation therapy. was positive for vimentin, NSE, TTF1, CEA but negative for GFAP. These results were compatible with cerebellar metastasis from a clear cell carcinoma. After surgery, the patient maintained a minimal static cerebellar syndrome. Brain imaging showed no residual tumor. No whole brain radiation was delivered. In order to detect the primary cancer, lung auscultation, thyroid examination as well as organs of abdomen examination and digital rectal exam were normal. Full blood counts were normal. Total body computed tomography (CT) was normal. Bronchofibroscopy revealed an inflammatory aspect of the left bronchi and its biopsy showed chronic inflammation. Positron emission tomography (PET) scan was not available in 2002. Tumor markers levels including PSA, CEA, CA19-9, and NSE were also normal. The patient was discharged with physical examination and CT every 4 months in the first year, then every six months during two years and then annually. In June 2014 , CT scan revealed a nonspecific $5 \mathrm{~mm}$ node in the right lung. Brain CT showed no recurrent disease (Fig. 2). Actually, our patient remains asymptomatic with no recurrent disease 14 years after the diagnosis of BM.

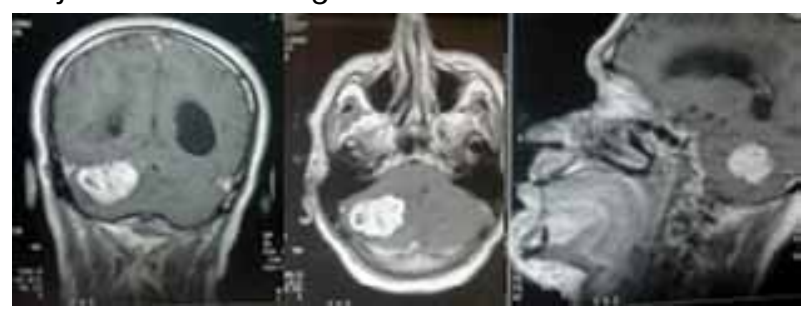

Fig. 1. Cerebral MRI: cerebellar metastasis

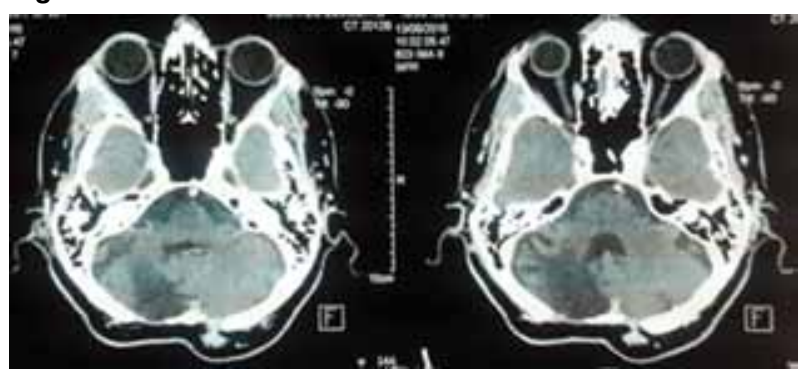

Fig. 2. Cerebral CT: cerebellar porencephalic cavity after surgery with no recurrence 


\section{DISCUSSION}

The definition of CUP includes patients with histological confirmed metastatic cancer in whom a detailed medical history, thorough physical examination and complete laboratory investigations fail to identify the primary site [2]. This is due to the fact that the presumed primary tumor is able to metastasize before becoming large enough to be identified [7]. BMUP represents $15 \%$ of all BM [4-6]. In about $50 \%$ of BMUP cases, primary lung cancer is found to be the most frequent primary site $(51 \%)$ [8]. Although the breast is the second most primary site in BM (10-17\%). In the reported cases of BMUP, melanoma represented $8 \%$, the pancreatic cancer was found in $8 \%$ and gastrointestinal cancers in $19 \%$ cases [9-12]. In our patient histopathological study with immunohistochemistry was compatible with cerebellar metastasis from a clear cell carcinoma. However, exploration did not find the primitive.

About $85 \%$ of BMUP are found in the cerebral hemispheres, $10-15 \%$ found in the cerebellum and $3 \%$ in the brainstem. In our case, the BM was located in the right cerebellum. Certain primary tumors such as those originating from kidney and colon are more likely to metastasize to the cerebellum.

As for the clinical presentation, the mean age of BMUP's patients ranges from 50 to 60 years with a male predilection. The predominant presenting symptom in BM is headaches as it is the case of our patient. Fifty percent of patients with BM have motor or language deficits and $10-15 \%$ of them present seizures [13]. Approximately, $10 \%$ of BM are asymptomatic and the diseases' spread is identified through routine imaging $[14,15]$.

The preferred modality for detecting brain lesions is MRI. Despite the accuracy of MRI, CT imaging is often used when MRI is either not available or not suitable for particular patients [16]. Despite the sensitivity of MRI, $11 \%$ of patients with a brain lesion are given a false-positive diagnosis of either a BM or a primary brain cancer [17-20].

The dilemma of distinguishing between a BM and a primary brain tumor can only be solved by biopsy or tumor resection with histopathological study [21, 22]. The choice between biopsy and surgical resection depends on many factors: number of brain lesions, its location, patient's general condition (Karnofsky performance status - KPS, age, comorbidity) and presence or not of accessible extra-cranial metastases. In our case, cerebral MRI showed a single lesion of the right cerebellum, so that he underwent a complete resection of the tumor.

In order to detect the primary cancer, physical examination was normal. Full blood counts were normal. Total body CT was normal. Bronchofibroscopy revealed an inflammatory aspect of the left bronchi and its biopsy showed chronic inflammation. PET scan was not available in 2002. Nowadays, the combination of PET/CT plays an important role to search the primitive in case of CUP $[23,24]$.
In our case, the immunohistochemical staining was positive for vimentin, NSE, TTF1, CEA but negative for GFAP. These results concluded to cerebellar metastasis from a clear cell carcinoma.

In the study of Drlicek et al. [25], 40 cases of BM from known primary tumors were blinded and reevaluated based on the immunohistochemical staining pattern. An established panel of antibodies was used including: CK AE1/AE3, CK7, CK20, CK10/13, CK18, vimentin, S100, TTF-1, surfactant, PSA, CA15-3, CA125, and CA19-9. This panel of 13 different antibodies was able to identify the primary in 29 out of 40 BM correctly (72.5\%). In this series, $1 / 11$ of lung cancer BM had a CK7 negative-CK20 negative profile.

The treatment of $\mathrm{BM}$ is an oncologic emergency. The recommendation of the subcommittee of the American Academy of Neurology (AAN) is that in patients with newly diagnosed brain tumor prophylactic anticonvulsants should not be used, it has to be initiated from the time of the first seizure [26]. For patients who undergo surgery, AAN recommends to stop them in 1 to 4 weeks postoperatively. All patients should have corticotherapy. Patients with single BM, good KPS and no disseminated disease, should have surgery or radiosurgery (if lesion less than $3 \mathrm{~cm}$ ) to achieve local tumor control. Radiosurgery is less invasive and gives the same result as with surgery but it does not provide tissue diagnosis. Postoperative whole brain radiation therapy (WBRT) helps to sterilize the other areas and improves survival [18]. In case of multiple BM, WBRT is given usually in doses of 30 Gy in 10 fractions or $20 \mathrm{~Gy}$ in 5 fractions. Chemotherapy has a relatively small role in case of BMUP. In fact, only few molecules could cross the blood-brain barrier.

Our patient was aged less than 65 years, he had a KPS $>70$, his BM was single and completely resected and finally, he had no other metastases. All these factors explain the good evolution and the long survival of this patient $[27,28]$.

In a retrospective study, the median overall survival for patients with a solitary BMUP was 7.3 vs 3.9 months for patients with multiple metastases $(p=0.05)$. Patients who underwent resection of BM before WBRT had a significant better overall survival than those who underwentWBRT alone (9.5 vs 3.6 months median survival, respectively) [15]. The majority of these patients die from extracranial disease and only a minority (15\%) will live more than five years [29]. To our knowledge, our patient represents the first case described in the literature with an overall survival exceeding 10 years.

\section{CONCLUSIONS}

BMUP is an ambiguous disease. It poses a problem for the oncological patient and his doctor. The prognosis of this entity is very poor. This case is exceptional; firstly given its rarity and secondly due to the long survival that exceeded 10 years. The conclusion that can be drawn is that within BMUP exist patients with a very good prognosis that must be collected and published in order to base recommendations.

Conflict of interest. None. 


\section{REFERENCES}

1. Giordana MT, Cordera S, Boghi A. Cerebral metastases as first symptom of cancer: a clinico-pathologic study. J Neurooncol 2000; 50: 265-73.

2. Pavlidis N, Khaled H, Gaafarb R. A mini review on cancer of unknown primary site: a clinical puzzle for the oncologists. J Adv Res 2015; 6: 375-82.

3. Polyzoidis KS, Miliaras G, Pavlidis N. Brain metastasis of unknown primary: a diagnostic and therapeutic dilemma. Cancer Treat Rev 2005; 31: 247-55.

4. Sheehan J, Niranjan A, Flickinger JC, et al. The expanding role of neurosurgeons in the management of brain metastases. Surg Neurol 2004; 62: 32-41.

5. Bender Al, Posner JB. Current treatment of brain metastases. In: Advanced techniques in central nervous system metastases. RJ Maciunas, ed: Parkridge: AANS, 1998: $1-15$.

6. DeAngelis LM, Gutin PH, Leibel SA, et al. Intracranial tumors. Diagnosis and treatment. 1st ed. London: Martin Dunitz, 2002.

7. Pavlidis H, Briasoulis E, Hainsworth J, Greco FA. Diagnostic and therapeutic management of cancer of an unknown primary. Eur J Cancer 2003; 39: 1990-2005.

8. Le Chevalier T, Smith FP, Caille P, et al. Sites of primary malignancies in patients presenting with cerebral metastases. A review of 120 cases. Cancer 1985; 56: 880-2.

9. Borgelt B, Gelber R, Kramer S, et al. The palliation of brain metastases: final results of the first two studies by the Radiation Therapy Oncology Group. Int J Radiat Oncol Biol Phys 1980; 6: 1-9.

10. Winston KR, Walsh JW, Fischer EG. Results of operative treatment of intracranial metastatic tumors. Cancer 1980; 45: 2639-45.

11. Yamada K, Miura M, Miyayama H, et al. Brain metastases from asymptomatic adenocarcinoma of the pancreas: an autopsy case report. Surg Neurol 2002; 58: 332-7.

12. Delattre JY, Krol G, Thaler HT, Posner JB. Distribution of brain metastases. Arch Neurol 1988; 45: 741-4.

13. Patchell RA. The management of brain metastases. Cancer Treat Rev 2003; 29: 533-40.

14. Nussbaum ES, Djalilian HR, Cho KH, Hall WA. Brain metastases. Histology, multiplicity, surgery, and survival. Cancer 1996; 78: 1781-8.

15. Bartelt S, Lutterbach J. Brain metastases in patients with cancer of unknown primary. J Neurooncol 2003; 64: $249-53$.
16. Pearl ML, Talgat G, Valea FA, Chalas E. Psychiatric symptoms due to brain metastases. Med Update Psychiatr 1998; 3: 91-4.

17. Posner JB. Management of brain metastases. Rev Neurol (Paris) 1992; 148: 477-87.

18. Patchell RA, Tibbs PA, Walsh JW, et al. A randomized trial of surgery in the treatment of single metastases to the brain. N Engl J Med 1990; 322: 494-500.

19. Jeong HJ, Chung JK, Kim YK, et al. Usefulness of whole-body (18)F-FDG PET in patients with suspected metastatic brain tumors. J Nucl Med 2002; 43: 1432-7.

20. Tosoni A, Ermani M, Brandes AA. The pathogenesis and treatment of brain metastases: a comprehensive review. Crit Rev Oncol Hematol 2004; 52: 199-215.

21. Schwartz KM, Erickson BJ, Lucchinetti C. Pattern of T2 hypointensity associated with ring-enhancing brain lesions can help to differentiate pathology. Neuroradiology 2006; 48: 143-9.

22. Law M, Cha S, Knopp EA, et al. High-grade gliomas and solitary metastases: differentiation by using perfusion and proton spectroscopic MR imaging. Radiology 2002; 222: 715-21.

23. Milović M, Popov I, Jelić S. Tumor markers in metastatic disease from cancer of unknown primary origin. Med Sci Monit 2002; 8: MT25-30.

24. Gutzeit A, Antoch G, Kühl H, et al. Unknown primary tumors: detection with dual-modality PET/CT - initial experience. Radiology 2005; 234: 227-34.

25. Drlicek M, Bodenteich A, Urbanits S, Grisold W. Immunohistochemical panel of antibodies in the diagnosis of brain metastases of the unknown primary. Pathol Res Pract 2004; 200: 727-34.

26. Glantz MJ, Cole BF, Forsyth PA, et al. Practice parameter: anticonvulsant prophylaxis in patients with newly diagnosed brain tumors. Report of the Quality Standards Subcommittee of the American Academy of Neurology. Neurology 2000; 54: 1886-93.

27. Hess KR, Abbruzzese MC, Lenzi R, et al. Classification and regression tree analysis of 1000 consecutive patients with unknown primary carcinoma. Clin Cancer Res 1999; 5: 3403-10.

28. Le Chevalier T, Cvitkovic E, Caille P, et al. Early metastatic cancer of unknown primary origin at presentation. A clinical study of 302 consecutive autopsied patients. Arch Intern Med 1988; 148: 2035-9.

29. Nguyen LN, Maor MH, Oswald MJ. Brain metastases as the only manifestation of an undetected primary tumor. Cancer 1998; 83: 2181-4. 